\title{
Pratiques
}

Linguistique, littérature, didactique

\section{Pratiques de 1974 à 2007 : présentation du classement des articles et premiers constats sur les évolutions de la revue}

Jean-Pierre Benoit

\section{(2) OpenEdition \\ Journals}

Édition électronique

URL : http://journals.openedition.org/pratiques/1162

DOI : $10.4000 /$ pratiques. 1162

ISSN : 2425-2042

Éditeur

Centre de recherche sur les médiations (CREM)

Édition imprimée

Date de publication : 15 juin 2008

Pagination : 235-247

Référence électronique

Jean-Pierre Benoit, «Pratiques de 1974 à 2007 : présentation du classement des articles et premiers constats sur les évolutions de la revue », Pratiques [En ligne], 137-138/2008, mis en ligne le 15 juin 2008, consulté le 02 mai 2019. URL : http://journals.openedition.org/pratiques/1162 ; DOI : 10.4000/ pratiques. 1162 


\section{Pratiques de 1974 à 2007 : présentation du classement des articles et premiers constats sur les évolutions de la revue}

\section{Jean-Pierre Benoit}

Université de Nantes, CREN, E A 2661, www.cren.net

Membre du collectif éditeur de Pratiques pendant vingt ans j'ai voulu revenir sur le travail accompli par la revue en établissant pour ce numéro dédié à l'un de ses fondateurs, Jean-François Halté, une bibliographie raisonnée du corpus constitué par les articles de Pratiques du numéro 1 de 1974 au numéro 135-6 de 2007. Ce corpus est très riche, mais le volume qu'il a pris et le nombre d'articles des numéros doubles de ces dernières années le rend difficile à utiliser pleinement, malgré les entrées déjà permises par le site www.pratiques-cresef.com. C'est pourquoi j' ai proposé un classement qui les complète. Il sera mis en ligne.

Dans ce numéro où l'espace est beaucoup plus limité je ne peux que présenter ce travail et proposer quelques premières analyses à partir de ce qu'il peut faire apparaître.

\section{Classer les articles de Pratiques, pourquoi et comment?}

On va expliciter les objectifs de ce travail, sa méthodologie dans la constitution du corpus et son classement, enfin donner et un extrait de ce classement.

\subsection{Objectifs du classement des articles}

Actuellement le site du CRESEF a deux entrées, l'une chronologique, par numéros, l'autre par auteurs, avec des liens hypertextes entre elles qui permettent de retrouver tous les articles de chaque auteur. Il est déjà riche, agréable et fort utile, mais un classement supplémentaire des articles devrait donner de nouvelles possibilités à ceux qui le consultent, qu'ils soient étudiants, professeurs stagiaires, enseignants, formateurs ou chercheurs.

Il s'agit de les aider à retrouver des ressources qu'ils n'auraient peut-être pas 
pu trouver ou pensé à chercher dans tel ou tel numéro. On espère que cet outil supplémentaire aidera à mieux révéler les ressources et les richesses de ce corpus et les points d'appui qu'elles peuvent donner - c'est particulièrement important dans le contexte éducatif et universitaire actuel - et à les utiliser davantage.

Il me semble aussi que les premières analyses de ce corpus proposées ici peuvent révéler à l'équipe de rédaction de la revue des lignes de force qui peuvent ne pas apparaître quand on est dans l'action et des champs sur lesquels revenir ou encore à explorer.

\subsection{Constitution du corpus et présentation des références}

Pour constituer le corpus je suis parti de la liste complète des articles par numéros, en format texte pour pouvoir la retravailler dans sa présentation, puis j'ai utilisé ce premier fichier chronologique pour coder les articles et constituer un second fichier texte, où chacun est classé cette fois par champs dont on trouvera les définitions au paragraphe suivant.

Déduction faite des présentations courtes de numéros, des notes de lecture et de certaines notes de conjoncture et tables rondes ou entretiens le corpus des articles parus dans Pratiques des années 1974 à 2007 incluse est constitué de près de 800 articles.

Comme nous envisagions une publication papier dans la revue j'ai visé à présenter les références dans une concision maximale, ce qui les rend différentes des normes bibliographiques habituelles : dans chaque catégorie elles sont données chronologiquement dans l'ordre des numéros avec indication de leur année, puis par article. Comme sur son site le nom de la revue, Pratiques, n'y est pas mentionné, mais les pages des articles restent indiquées pour permettre d'y aller directement. Pour les auteurs, on donne leurs prénoms (en initiales ici) puis leurs noms avec les liens hypertextes qui renvoient à la liste des articles qu'ils ont publiés dans la revue. Cela se présente ainsi :

...103 texte et discours

$n^{\circ} 6(1975)$

B. Combettes, J. Fresson «Quelques éléments pour une linguistique textuelle», pp. 25-55. n'11-12 (1976)

M. Charolles «Grammaire de texte - théorie du discours - narrativité », pp. 133-154.

B. Minel «Pour une théorie des pratiques discursives », pp. 155-190.

$n^{\circ} 13(1977) \ldots$

Dans cet exemple, il y a deux articles dans le numéro 11-12, ce qui permet de les regrouper. Malgré tout ce travail pour gagner de la place dans leur présentation, de petites marges et des références en police 10 les deux fichiers texte représentent chacun une trentaine de pages environ. C'était trop pour une publication dans la revue, même en plusieurs fois... Elle sera donc faite sur le site www.pratiques-cresef.com qui est moins limité en place, qui peut être tenu à jour et qui permet d'utiliser les liens hypertexte.

Le corpus et sa présentation définis explicitons les critères de classement des articles. 


\subsection{Critères de classement}

Des études sur la transmission des savoirs académiques et didactiques dans plusieurs IUFM à travers les références utilisées dans les mémoires professionnels de professeurs stagiaires de Lettres m'avaient permis de mettre au point des grilles de classement de ce type de données (Benoit 1998 et 2004). Je suis parti d'elles pour classer les articles et nous avons fait le choix de fonder ce classement sur la distinction entre didactiques « restreinte » et «élargie» ${ }^{(1)}$.

Par didactique « restreinte » on entend celle des champs qui correspondent aux disciplines universitaires de référence à la discipline scolaire lettres-français langue première : la langue, codes 1 et dérivés, la lecture, codes $2 . . .$, l'oral, codes $3 \ldots$, l'écriture, codes 4.

Pour chacun de ces champs on a réparti les articles en études théoriques et descriptives - codes $\mathrm{x} 0 \ldots$ - et en propositions didactiques, codes $\mathrm{x} 1 \ldots$ On a prévu une section 5 pour indexer les articles qui étudient ou proposent des interactions entre les quatre champs définis précédemment ou d'autres interactions.

En didactique «élargie » on a placé les articles qui sont centrés sur l'apprentissage, éclairé par les « sciences humaines applicables à l'enseignement »-dimensions psychologiques (6), sociologiques, socioculturelles et anthropologiques (7), pédagogico-didactiques (8) ou historiques (9). On y a mis aussi les comparaisons internationales ou avec d'autres disciplines (10), les commentaires sur les politiques éducatives et les programmes contemporains et les propositions (11), les analyses de matériels d'enseignement contemporains (12) et enfin les articles sur le recrutement et la formation des professeurs de français langue première (13).

Le codage a permis d'affiner ces catégories, ce qui donne le plan de classement suivant, qui devrait permettre aux lecteurs de la revue de se retrouver plus facilement dans le fichier qui sera mis sur le site.

1 LANGUE

\section{Didactique « restreinte » du français langue première}

10 ANALYSES DE LA LANGUE

100 points généraux

101 lexique

102 morpho-syntaxe et phrase

103 texte et discours

104 orthographe, lexicale et grammaticale

11 DIDACTIQUE DE LA LANGUE

110 points généraux

111 didactique du lexique

112 didactique de la morpho-syntaxe et de la phrase

113 didactique du texte et des discours

114 didactique de l'orthographe

(1) Un autre classement qui pourrait s'y ajouter a été envisagé mais n'a pas été fait ici, d'autant qu'il recoupe partiellement la distinction entre les articles théoriques et didactiques, bien qu'il y ait une didactique non praxéologique... il s'agit d'un classement par visées, praxéologique ou non, La mise sur site permettra peut-être de le réaliser. 


\section{LECTURE}

\section{POINTS GÉNÉRAUX SUR LA LECTURE}

201 La lecture, définitions et stratégies linguistiques et cognitives

202 Registres, tons, postures

\section{ANALYSES D'CEUVRES LITTÉRAIRES}

$\mathbf{2 1 0}$ points généraux pour l'analyse d'œuvres littéraires

211 analyses d'œuvres narratives ou du genre narratif

\section{Fictions}

2112 récits de vie et (auto) biographies

212 analyses d'œuvres théâtrales ou du genre théâtral

213 analyses d'œuvres poétiques ou du genre poétique

214 analyses d'images

215 analyses de paralittératures

216 analyses de «littérature de jeunesse »

217 analyse d'œuvres littéraires argumentatives (« littérature d'idées », « apologue »...)

22 ANALYSES DE TEXTES ET DE DISCOURS NON LITTÉRAIRES

220 points généraux

221 presse

222 écrits documentaires et scientifiques

223 communication politique ou publicitaire

224 consignes

225 divers

226 supports numériques, hypertextualité numérique

23 DIDACTIQUE DE LA LECTURE

230 points généraux

231 didactique de lectures littéraires

2310 en facteur commun

2311 didactique de lectures d'œuvres narratives littéraires

2312 didactique de lectures d'œuvres théâtrales

2313 didactique de lectures d'oeuvres poétiques

2314 didactique de la lecture d'images fixes ou mobiles

2315 didactique de paralittératures

2316 didactique de lectures de "littérature de jeunesse »

2317 didactique de lectures d'œuvres littéraires et argumentatives ( littérature d'idées», « apologue »...)

2318 didactique des lectures de textes et discours non littéraires

Publicité

Documentaires

Consignes

Presse

Autres

3 ORAL

30 ETUDES DE L'ORAL

31 DIDACTIQUE DE L'ORAL

32 JEU THÉÂTRAL

4 ÉCRITURE

40 ÉCRITURE

41 DIDACTIQUE DE L'ÉCRITURE

410 points généraux

411 didactique d'écrits de fiction

4101 Ecritures narratives

écritures de fictions 


\section{écritures biographiques}

écritures de paralittératures

4102 Ecritures théâtrales

412 didactique d'écrits « ancrés dans la situation d'énonciation "

413 didactique des écrits scolaires et universitaires

5 INTERACTIONS

50 INTERACTIONS ENTRE LECTURE ET ÉCRITURE...

51 INTERACTIONS ENTRE ORAL ET LECTURE/ ÉCRITURE

52 INTERACTIONS ENTRE LANGUE ET LECTURE/ÉCRITURE...

53 INTERACTIONS ENTRE LANGUE ET ORAL

54 INTERACTIONS ENTRE LECTURE-ÉCRITURE ET HISTOIRE LITTÉRAIRE

55 INTERACTIONS AVEC D'AUTRES DISCIPLINES

56 INTERACTIONS ENSEIGNANTS ELEVES

57 INTERACTIONS ENTRE ELEVES

58 INTERACTIONS ENTRE ELEVES ET ORDINATEUR

\section{Didactique « élargie » du français langue première}

\section{DIMENSIONS PSYCHOLOGIQUES}

7 DIMENSIONS SOCIOLOGIQUES, SOCIOCULTURELLES ET ANTHROPOLOGIQUES, RAPPORTS AUX SAVOIRS,

AU LANGAGE...

8 DIMENSIONS PÉDAGOGICO-DIDACTIQUES

9 HISTOIRE DE LENSEIGNEMENT ET DE LA DISCIPLINE : INSTITUTIONS ÉDUCATIVES, PROGRAMMES, MANUELS ANCIENS...

\section{COMPARAISONS}

101 comparaisons internationales

102 comparaisons avec d'autres disciplines

11 POLITIQUES ÉDUCATIVES ET PROGRAMMES CONTEMPORAINS, COMMENTAIRES ET PROPOSITIONS

12 MATÉRIELS D'ENSEIGNEMENT CONTEMPORAINS

13 RECRUTEMENT ET FORMATION DES ENSEIGNANTS DE FRANÇAIS LANGUE PREMIÈRE

Pour classer les articles dans ces catégories, on a consulté ce site, repris la collection et utilisé les résumés et mots clés donnés à partir du numéro 90. Pour pouvoir espérer publier cette bibliographie raisonnée et établir des statistiques sur la répartition des articles on a du se résoudre à ne placer ceux-ci qu'à un seul endroit, au besoin dans la section 5 interactions... Le choix d'une dominante n'a pas toujours été facile et il reste contestable. Peut-être ce classement pourra-t-il être enrichi sur le site ? Je suis en tout cas prêt à en préparer la «maintenance » en rentrant les demandes de modifications que me feront des auteurs qui estimeront que tel ou tel de leurs articles serait mieux placé ailleurs, en affinant les catégories en fonction des remarques qui me seraient faites et en actualisant mes deux fichiers, chronologique et classé avec les articles des années prochaines... ${ }^{(*)}$

Le corpus étudié et les modalités de son classement définis, donnons-en quelques premières analyses.

\section{Premières analyses du corpus}

Ces premières remarques ne demandent qu'à être affinées par des chercheurs qui étudieront, on l'espère à partir de ce classement, ce corpus de première im-

(*) Courriel : jean-pierre.benoit@univ-nantes.fr 
portance pour la naissance et le développement de la didactique du français langue première.

Voyons ce que le catalogue chronologique tel qu'il existe sur le site fait déjà apparaître, puis ce que les occurrences classées manifestent.

\subsection{Ce que le site faisait déjà apparaître}

Les balayages des sommaires et des présentations des numéros que l'on peut visualiser sur le site permettent déjà de formuler diverses remarques. On pourrait en faire sur les arrivées ou périodes d'activité des auteurs mais on se limitera ici à l'évolution de la revue...

Sa périodicité est d'abord irrégulière avant de se stabiliser comme trimestrielle avec 128 pages de 1980 à 1998, puis comme semestrielle à 256 .

Son format est stabilisé dès la deuxième livraison, mais la couverture change plusieurs fois : les titres et sommaires sont noirs sur fond rouge en 1974, puis de couleurs claires sur fond blanc jusqu'à 1979. En 1980 le sommaire disparaît de la couverture, qui est illustrée dans sa partie supérieure de dessins originaux, à obtenir chaque fois, jusqu'en 1988. C'est avec le numéro 58 que la présentation actuelle apparaît, un fond à nuances de gris souligné discrètement par une couleur. Elle garde le logo introduit en 1980, entouré du triptyque de 1974 « Théorie, Pratique, Pédagogie » qui devient en 2007 «Linguistique, Littérature, Didactique », vingt ans après l'apparition d'Enjeux, la première revue qui se soit définie sur sa couverture comme « revue de didactique du français » à un moment où l'expression a suffisamment émergé, mouvement confirmé par la parution en 1992 du Que sais-je? de Jean-François Halté, La didactique du français : les membres du collectif de Pratiques, ont joué un rôle déterminant dans cette émergence mais quand ils ont lancé et affirmé la revue ils ignoraient que ce qu'ils défendaient et illustraient s'appellerait didactique du français langue maternelle puis première...

Les sommaires donnés sur le site montrent encore que c'est à partir du numéro 90 de 1996 que des résumés des articles sont donnés, mais pour aller plus loin il faut reprendre la collection.

\subsection{D'autres remarques en feuilletant les numéros}

Il faut disposer de la collection pour pouvoir préciser que c'est aussi dans ce numéro 90 qu'un comité de lecture s'ajoute au collectif, à l'origine de trois personnes, Jean-François Halté, Raymond Michel et André Petitjean, qui s'est progressivement étendu à une douzaine de membres et qui s'est stabilisé. Dans le numéro 133-134 de 2007 il est remanié, comme le collectif.

Des présentations de numéros explicitent ces évolutions de la revue. On en citera deux, l'éditorial du premier et celle du 97-98, de 1998.

Le premier éditorial définit «l'intervention de la revue». En correspondance avec la couleur rouge de sa couverture il est très politique ; on peut y lire, page 3 «L'école, telle que nous la souhaitons (démocratique) ne sera vraiment telle que lorsque la société aura changé (socialisme), ce qui ne signifie nullement qu'il faille attendre ce moment pour agir en/sur elle. » (p. 3).

Vingt-cinq ans plus tard, la présentation du $\mathrm{n}^{\circ}$ 97-98, La transposition didactique en français (Petitjean 1998), fait un bilan du chemin parcouru et annonce 
que «Pratiques va tendanciellement changer de nature », parce que l'époque, le lectorat et le collectif ont changé, ses membres s'étant spécialisés et ayant eu à diversifier leurs activités. Elle annonce que Pratiques aura des problématiques «moins praxéologiques que théoriques, historiques et critiques », plus « méta », pour « chercher à élucider les causes des échecs et réussites et transformer le fonctionnement effectif des classes au profit des élèves et de leurs enseignants ».

Pour approfondir l'analyse du « corpus Pratiques » passons à celle des occurrences de son classement.

\subsection{Ce que les occurrences du classement manifestent}

Dix ans déjà depuis cette présentation et Pratiques aura 35 ans 1'année prochaine... Avec une telle longévité il ne suffit pas d'analyser globalement le classement de ses articles. On propose donc d'affiner son étude en les répartissant par périodes. On peut penser que nous entrons dans une nouvelle phase, marquée à l'interne par le remaniement récent de l'équipe éditoriale et à l'externe par l'actuelle tentative de restauration de "l'ancienne configuration didactique » que la revue a beaucoup critiquée...

De 1974 à 2007 il me semble que l'on peut déterminer trois périodes de Pratiques, à la fois à partir de critères internes et d'éléments externes.

Les critères internes sont les évolutions matérielles qui viennent d'être évoquées. Les éléments externes à prendre en compte peuvent être l'influence de la revue et les contextes politiques qui ont rapidement en France des effets sur l'enseignement de la langue nationale, décidément très idéologique ${ }^{(2)}$.

La première de ces trois périodes irait jusqu'en 1985, avec deux phases : fondée au début du septennat giscardien Pratiques critique vivement l'obsolescence des savoirs enseignés alors et entreprend de les renouveler en théorisant, expérimentant et diffusant ce qu'elle appelle " grammaire du texte », puis « linguistique textuelle», qui devient une rubrique dans le numéro de janvier 1976 au moment où Bernard Combettes, membre du collectif, publie une collection de manuels fondateurs pour le collège, Bâtir une grammaire - le titre manifeste qu'il s'agit plutôt d'en faire que de la mémoriser - et De la phrase au texte. L'alternance politique de 1981 permet des réflexions comme celles de la commission Chevalier, où des membres du collectif ont des responsabilités dans les groupes collège et lycée, et cette première période se clôt par les programmes de 1985 qui résultent des travaux de cette commission : on y reconnaît en effet des apports du collectif comme la Pratique raisonnée de la langue « finalisée » pour la lecture et la production orale ou écrite et le dépassement des limites de la phrase - la situation de discours, l'auteur et le destinataire d'un message, les personnes, l'implicite, les « types de textes »...

La deuxième période de Pratiques irait de 1986 jusqu'à 1997 : les linguistiques textuelle puis discursive se diffusent alors rapidement dans l'enseignement et la didactique du français y émerge.

(2) Ne pouvant développer ici je renvoie à mon historique détaillé des deux premières périodes pages 22 à 45 de "L'émergence du discours et de l'argumentation dans le champ de la didactique du français. Les "savoirs savants" et leurs transpositions dans les textes officiels depuis 1975 ", in L'émergence du discours dans l'enseignement du français, J.-P. Benoit éd. ; CRDP de Rennes, collection Lettres ouvertes (11-59). 
La troisième période commencerait avec les annonces du numéro 97-98 ${ }^{(3)}$ et elle irait plutôt jusqu'à 2006 qu'à 2007 qui marque peut-être le début d'une quatrième époque, il faudra du recul pour le confirmer.

Ces trois périodes de Pratiques définies voici la double répartition, thématique et chronologique, des articles

\subsubsection{Tableau de répartition des articles}

On suit le plan de classement en donnant le total des occurrences 1974-2007 puis le détail de celles-ci par périodes - elles sont du même ordre, entre 278 et 252 articles par période. En gras les plus élevées. On a ajouté à gauche sous les titres, hors des colonnes réservées à ces occurrences afin de ne pas les fausser, des totalisations des sous-sections qui suivent, ainsi que quelques pourcentages, globaux et par périodes des occurrences par rapport au total global ou à ceux des trois périodes.

\section{Champs}

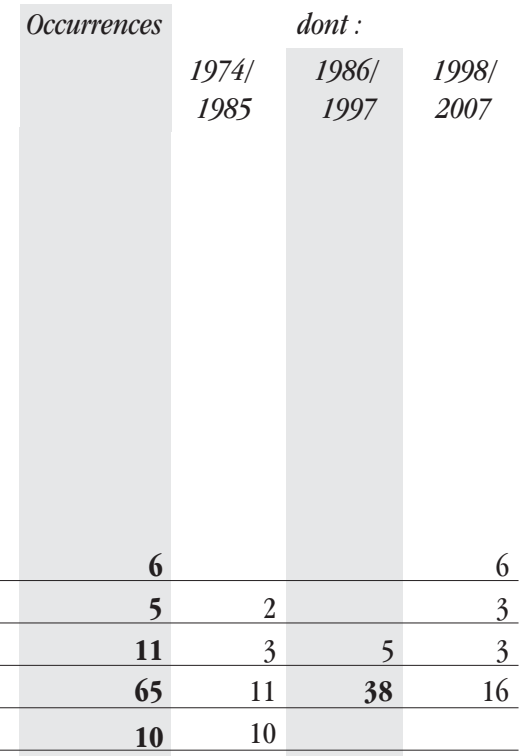

11 d. de la langue

(T 52 articles dont $\mathbf{2 7}, 16,9)$

$\%$ respectifs par rapport aux totaux

$\mathbf{6 , 6}, \mathbf{9 , 7}, 6,3$, et 3,6

\begin{tabular}{lrrrr}
110 points généraux & $\mathbf{1 3}$ & 7 & 1 & 5 \\
\hline 111 didactique du lexique & $\mathbf{6}$ & 4 & 2 & \\
\hline 112 didactique de la morpho-syntaxe & $\mathbf{5}$ & & 2 & 3 \\
\hline 113 didactique du texte et des discours & $\mathbf{1 8}$ & 7 & $\mathbf{1 0}$ & 1 \\
\hline 114 didactique de l'orthographe & $\mathbf{1 0}$ & 9 & 1 & \\
\hline
\end{tabular}

(3) Bernard Combettes et Michel Charolles relativisent alors les frontières entre la phrase et « le texte ou discours» dans «Contribution pour une histoire récente de l'analyse du discours », Langue française de mars 1999. 


\section{Lecture}

(T 205 articles 94, 64, 47)

$\%$ respectifs par rapport aux totaux

26, 33,8, 25, et 18,7

20 points généraux sur la lecture

(sous total $14 \mathrm{t}$ articles dont 5, 7,2)

$\%$ respectifs par rapport aux totaux

$1,7,1,8,2,7$, et 0,8

201 lecture, défs et stratégies

9

202 registres, tons, postures

5

2

\section{1 analyse d'œuvres littéraires}

(sous total $\mathbf{7 5}$ articles dont 39, 20, 16)

$\%$ respectifs par rapport aux totaux

9,6, 14, 7,8, et 6,3

210 points généraux pour l'analyse d'œuvres littéraires

13

211 œuvres ou du genre narratif

2111 fictions

\begin{tabular}{llll}
19 & 13 & 3 & 3 \\
\hline
\end{tabular}

2112 (auto) biographies

2

212 œuvres ou genre théâtral

213 œuvres ou genre poétique

214 images

215 paralittératures

$2 \quad 2$

216 « littérature jeunesse »

217 œuvres littéraires argumentatives

\section{2 analyse de textes et discours non littéraires}

(sous total 32 arrticles dont 13, 8, 11)

$\%$ respectifs par rapport aux totaux

$4,4,7,3$, et 4,3

220 points généraux

\begin{tabular}{lrrrr}
\hline 221 presse & $\mathbf{1 6}$ & 5 & 7 & 4 \\
\hline 222 écrits documentaires et scientifiques & $\mathbf{5}$ & 2 & 1 & 2 \\
\hline 223 communication pol ou publi & $\mathbf{6}$ & 5 & & 1 \\
\hline 224 consignes & $\mathbf{1}$ & & & 1 \\
\hline 225 divers & $\mathbf{1}$ & 1 & & \\
\hline 226 supports numériques & $\mathbf{3}$ & & & 3 \\
\hline $\mathbf{2 3}$ didactique de la lecture & & & & \\
$\begin{array}{l}\text { (sous total 84 articles dont 37, 29 18) } \\
\text { \% respectifs par rapport aux totaux }\end{array}$ & & & & \\
$\mathbf{1 0 , 7 , 1 3 , 3}, 11,3$, et 7 & & & & \\
230 points généraux & & & & \\
231 lectures littéraires & $\mathbf{1 0}$ & 7 & 2 & 1 \\
\hline 2310 points généraux & & & & \\
\hline 2311 d de 1 d'œuvres narratives littéraires & $\mathbf{1 1}$ & 1 & & 3 \\
\hline 2312 d de 1 d'œuvres théâtrales & $\mathbf{1 1}$ & 5 & 2 & 4 \\
\hline 2313 d de 1 d'œuvres poétiques & $\mathbf{1 1}$ & 6 & 5 & \\
\hline
\end{tabular}




\begin{tabular}{|c|c|c|c|c|}
\hline $2314 \mathrm{~d}$ de 1 d'images & 10 & 9 & 1 & \\
\hline 2315 d de paralittératures & 2 & & 2 & \\
\hline 2316 d de 1 de « littérature de jeunesse » & 7 & 1 & 5 & 1 \\
\hline $2317 \mathrm{~d}$ de 1 d'œuvres littéraires argumentatives & 2 & & & 2 \\
\hline $2318 \mathrm{~d}$ des 1 non littéraires & 16 & 5 & 6 & 5 \\
\hline $\begin{array}{l}\mathbf{3} \text { Oral } \\
\text { (T } \mathbf{2 6} \text { articles dont } \mathbf{1 8}, 0,8) \\
\% \text { respectifs par rapport aux totaux } \\
\mathbf{3 , 3}, \mathbf{6 , 5}, 0 \text { et } 3,2\end{array}$ & & & & \\
\hline 30 l'oral & 11 & 7 & & 4 \\
\hline 31 didactique de l'oral & 12 & 8 & & 4 \\
\hline 32 le jeu théâtral & 3 & 3 & & \\
\hline
\end{tabular}

\section{Ecriture}

(T 169 articles dont 36, 74, 59)

$\%$ respectifs par rapport aux totaux

21,6, 12,9, 28,9 et 23,4

\section{0 l'écriture}

41 didactique de l'écriture

(sous total $\mathbf{1 6 4}$ articles dont 36, 71, 57)

$\%$ respectifs par rapport aux totaux

20,9, 12,9, 27,7 et 22,6
410 points généraux

411 didactique d'écrits de fiction

4101 écritures narratives

Fictions

Biographies

Paralittératures

4102 écritures théâtrales

4103 écritures poétiques

412 didact d'écrits « ancrés »

413 didact d'écrits scolaires

5 Interactions

(T 75 articles : 18, 17, 30)

$\%$ respectifs par rapport aux totaux

9,6, 6,5, 6,6, et 11,9

50 i lecture/écriture...

51 i oral-lecture/ écriture

52 i langue-lecture/écriture...

53 i langue/oral

54 i lecture-écriture et histoire littéraire

$55 \mathrm{i}$ avec autres disciplines

56 i enseignants élèves

57 i entre élèves

58 i entre élèves et ordinateur

5

3

2

47

6

20

21

\section{;}


Total didactique « restreinte "

(T 614 articles dont 219, 214 et 181)

$\%$ respectifs par rapport aux totaux

$\mathbf{7 8 , 3}, 78,8, \mathbf{8 3}, \mathbf{6}$ et 71,8

DIDACTIQUE « ÉLARGIE »

6 Dimensions psychologiques

$\%$ respectifs par rapport aux totaux

0,4, 0,7, 0,4, et 0

7 Dimensions sociales, socio-cult., anthropologiques...

48

21

13

$\%$ respectifs par rapport aux totaux

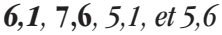

8 Dimensions pédagogiques

$\%$ respectifs par rapport aux totaux

$\mathbf{3 , 3}, \mathbf{6 , 1}, 2,3$, et 1,2

\section{Histoire}

\% respectifs par rapport aux totaux

$\mathbf{1 , 5}, 0,3,0,7$, et $\mathbf{3 , 6}$

\section{Comparaisons}

(T 18 articles : 0, 3, 15)

$\%$ respectifs par rapport aux totaux

2,3, 0, 1,2 et 6

101 comparaisons internationales

102 comparaisons avec d'autres disciplines

\begin{tabular}{lll}
7 & 2 & 5 \\
\hline
\end{tabular}

11 Politiques éducatives et programmes

$\%$ respectifs par rapport aux totaux

$\mathbf{4 , 7}, 3,6,2,7$, et 7,9

\section{Matériels d'enseignement}

$\%$ respectifs par rapport aux totaux

1, 1,4, 1,9 et 0,7

13 Recrutement/formation des enseignants de français langue première

$\%$ respectifs par rapport aux totaux

$2,1,1,4,1,9$, et 3

\section{Total didactique « élargie "}

(T 170 articles dont 59, 42 et 71)

$\%$ respectifs par rapport aux totaux

$21,7,21,2,16,4$ et $\mathbf{2 8 , 2}$ 


\subsubsection{Premiers commentaires}

On n'a pas la place ici de développer toutes les analyses que l'on pourrait tirer de ce tableau. A chacun donc d'y chercher ce qui l'intéresse ou le concerne le plus et d'en tirer les conclusions ou les conséquences, s'il participe à l'édition de la revue... Contentons-nous de quelques remarques les unes globales, sur 33 années de Pratiques et d'autres particulières, sur chaque période.

Sur l'ensemble de la parution 1974-2007, les 784 articles classés ici, 614 traitent des quatre champs de la « didactique restreinte » du français, la langue, la lecture, l'oral et l'écriture, ainsi que leurs interactions. Ils représentent plus de $78 \%$ du corpus. Avec $26 \%$ des articles c'est la lecture qui a fait 1'objet du plus grand nombre d'entre eux, puis l'écriture avec 21,6 et la langue avec 19.

Globalement toujours, en « didactique élargie » Pratiques a toujours beaucoup plus pris en compte les dimensions sociales, socio-culturelles voire anthropologiques de l'enseignement-apprentissage du français langue première (7) que ses dimensions psychologiques (6). C'est en cohérence avec son projet initial...

Chaque période d'environ dix ans a ses particularités :

Dans la première décennie les articles consacrés à la lecture et à sa didactique sont plus présents qu'ils le seront ensuite - près de $34 \%$. Dans une moindre mesure c'est aussi le cas de ceux sur l'oral et, en « didactique élargie » des articles » pédagogiques » $(8)$.

La période 1986-1997 est marquée par le doublement des articles sur l'écriture, essentiellement de sa didactique - ils atteignent près de $29 \%$, devant la lecture, passée à 25 et la forte augmentation des articles d'analyses linguistiques 43 au lieu de 26.

La dernière décennie est marquée par des rééquilibrages : en « didactique restreinte » les articles consacrés à la lecture et à sa didactique continuent à diminuer, passant à moins de $19 \%$ et ceux sur la langue à moins de 15 ; ceux sur l'écriture diminuent aussi de 4\%, mais ceux sur les interactions entre ces champs et, nouveauté, avec d'autres éléments augmentent sensiblement, passant de $6,5 \%$ à presque 12. L'ensemble des articles de « didactique élargie » passe de 16,4\% à plus de 28 , avec un développement particulier de l'histoire de la discipline (9), de la formation des professeurs de la discipline (13), et surtout de comparaisons, notamment avec d'autres disciplines (10) et d'études des politiques éducatives et des programmes (11). Comme l'avait annoncé la présentation du n97-98, La transposition didactique en français (Petitjean 1998), Pratiques a traité des problématiques « moins praxéologiques que théoriques, historiques et critiques, plus « méta ».

\section{Et maintenant ?}

On espère que le classement des articles de Pratiques par dominantes des points traités qu'on a proposé et que les regards rétrospectifs, mais pas nostalgiques, qu'il permettent seront utiles à ceux qui lisent la revue et à ceux qui la réalisent.

Les lecteurs reviendront peut-être plus commodément à la collection - à eux de l'utiliser au mieux... A ceux qui réalisent la revue de se servir de ces éclairages pour continuer à la faire vivre... 


\section{Références}

BENOIT, J.-P. (1998) : «Valeurs et savoirs en fin de formation initiale : analyse de mémoires professionnels de Lettres ", $7^{\mathrm{e}}$ colloque de l'AIDFLM, Bruxelles 16-19 septembre 1998, pp. 261-264.

- (2004) : «Cultures et pratiques professionnelles des stagiaires de Lettres et de leurs formateurs. Evolutions sur dix ans et spécificités locales », in Le mémoire professionnel en IUFM: bilans de recherches questions vives, Annette Gonnin-Bolo et Jean-Pierre Benoit eds. « Horizons pour la formation », INRP.

Petitjean, A, (1998) : «Pratiques a 25 ans... », Pratiques n97-98, pp. 3-5. 